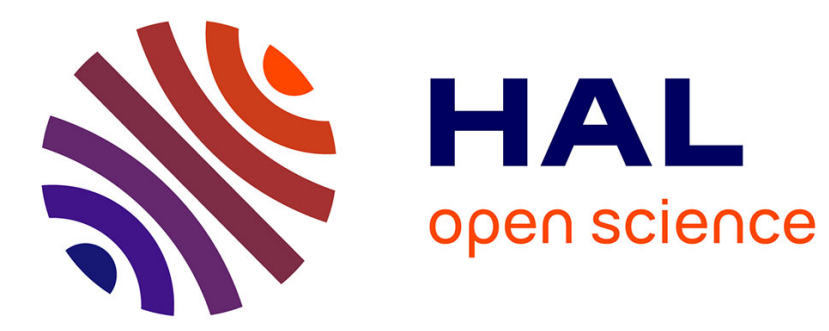

\title{
Modelling electric field gradients of icosahedral quasicrystals
}

\author{
Martin Klanjsek, Janez Dolinsek
}

\section{To cite this version:}

Martin Klanjsek, Janez Dolinsek. Modelling electric field gradients of icosahedral quasicrystals. Philosophical Magazine, 2005, 86 (03-05), pp.413-418. 10.1080/14786430500263959 . hal-00513585

\section{HAL Id: hal-00513585 \\ https://hal.science/hal-00513585}

Submitted on 1 Sep 2010

HAL is a multi-disciplinary open access archive for the deposit and dissemination of scientific research documents, whether they are published or not. The documents may come from teaching and research institutions in France or abroad, or from public or private research centers.
L'archive ouverte pluridisciplinaire HAL, est destinée au dépôt et à la diffusion de documents scientifiques de niveau recherche, publiés ou non, émanant des établissements d'enseignement et de recherche français ou étrangers, des laboratoires publics ou privés. 


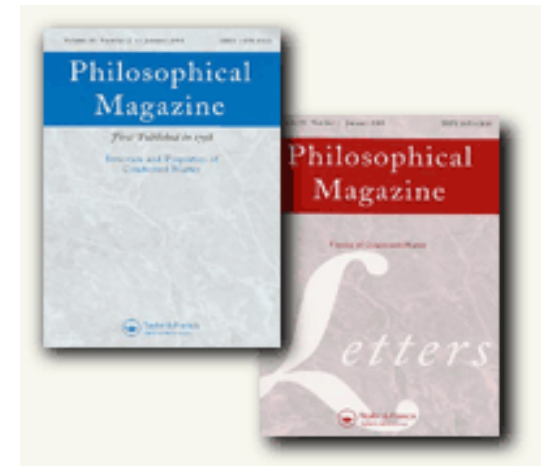

\section{Modelling electric field gradients of icosahedral quasicrystals}

\begin{tabular}{|r|l|}
\hline Journal: & Philosophical Magazine \& Philosophical Magazine Letters \\
\hline Manuscript ID: & TPHM-05-May-0147.R1 \\
\hline Journal Selection: & Philosophical Magazine \\
\hline $\begin{array}{r}\text { Date Submitted by the } \\
\text { Author: }\end{array}$ & 14-Jul-2005 \\
\hline Complete List of Authors: & $\begin{array}{l}\text { Klanjsek, Martin; Jozef Stefan Institute, Condensed Matter Physics } \\
\text { Department } \\
\text { Dolinsek, Janez; J. Stefan Institute, F5 }\end{array}$ \\
\hline Keywords: & quasicrystals, magnetic resonance \\
\hline Keywords (user supplied): & electric field gradient \\
\hline
\end{tabular}

\section{(5) ScholaroNE \\ Manuscript Central}




\begin{abstract}
A simple auxiliary model is presented that enables extracting general features of the EFG tensor distribution in icosahedral qusicrystals directly from the orientation dependence of the quadrupoleperturbed NMR spectrum. The only assumption of the model is a macroscopic icosahedral symmetry of the sample under study. It is shown that the model serves as a good filter since on the basis of the measured NMR spectrum orientation-dependence it can directly differentiate between qualitatively different icosahedral distributions of the EFG tensor orientations.
\end{abstract}

Keywords: Quasicrystals; Nuclear magnetic resonance; Electric field gradient

\title{
1 Introduction
}

In spite of the outstanding progress in the production of high-quality thermodynamically stable quasicrystals, the task of solving the quasicrystalline structures turned out to be an extremly difficult one [1,2]. Through X-ray and neutron diffraction experiments, the location of the majority of atoms can be successfully determined. However, as a finite number of diffraction peaks is accessible only, structure refinements lead to split atoms and atoms whose chemical identity cannot be determined [3]. One way to overcome this difficulty is by means of periodic approximants [4]. On the basis of diffraction patterns combined with ab initio calculations, an exact periodic model structure can be obtained [5,6], which can then be further optimized by means of numerical methods [7]. Examples include Cockayne model of icosahedral AlCuFe quasicrystal [5] and Quandt-Elser model of icosahedral AlPdMn quasicrystal [6], the first one with 128 and the second one with 65 atoms per unit cell. Due to their tractable size, such models are convenient for the calculations of physical quantities or their distributions, which can then be compared to the results of proper experiments in order to test the adequacy of the models. One such quantity is the electric field gradient (EFG) tensor at the sites of atomic nuclei. An information on its distribution over atomic sites of the same species can be accessed via spectroscopic techniques such as nuclear magnetic resonance (NMR) [8,9], nuclear quadrupole resonance (NQR) [10] and Mössbauer spectroscopy [7,11].

In the standard procedure of testing an agreement between the structural model and the experiment, the structural model with some degrees of freedom is taken as a starting point. The degrees of freedom usually include shifts of positions of single atoms or exchanges within some pairs of atoms of different species [7]. While the degrees of freedom are being varied, the distribution of the EFG tensors is numerically calculated in every step and the results are compared to the experiment. However, the procedure can be reversed. Directly from the results of the experiment some general 
features of the EFG tensor distribution can be extracted, which can, in turn, help in deciding how to vary the degrees of freedom of the structural model. For the reversed procedure to be feasible, the extraction of the general features should proceed via a simple auxiliary model with as few assumptions as possible. In this paper we present a simple auxiliary model suitable for the extraction of the EFG tensor distribution in icosahedral quasicrystals. The model is based on the macroscopic icosahedral symmetry of the sample under study, this being the only assumption involved. It states that in addition to the atomic site with the EFG tensor $\mathbf{V}$, a set of atomic sites should be found, whose EFG tensors are given by $\mathbf{V}_{i}=\mathbf{R}_{i}^{-1} \mathbf{V} \mathbf{R}_{i}\left(i=2, \ldots, 120 ; \mathbf{R}_{1}=\mathbf{I}\right)$, where $\mathbf{R}_{i}$ are the elements of an icosahedral point group, the power of which amounts to 120. In other words, every EFG tensor has 119 partners that can be arrived at by applying group elements to the original tensor. The model so designed still possesses plenty of degrees of freedom since an arbitrary number of the initial EFG tensors could be chosen, which are then multiplied in accordance with the icosahedral symmetry.

\section{NMR spectrum}

An observable that leads to the distribution of the EFG tensors in a macroscopic sample is quadrupoleperturbed ${ }^{27} \mathrm{Al}$ NMR frequency. In the absence of perturbing interactions, $2 I+1$ Zeeman levels of the ${ }^{27} \mathrm{Al}$ nuclear spin $(I=5 / 2)$ are equally spaced and there is only one NMR frequency, the Larmor frequency $v_{\mathrm{L}}$. Though quadrupole interaction is strong, it represents a perturbation to the main Zeeman interaction and affects the central transition $-1 / 2 \leftrightarrow 1 / 2$ in second order only, while it affects the remaining satellite transitions, i.e. $\pm 5 / 2 \leftrightarrow \pm 3 / 2$ and $\pm 3 / 2 \leftrightarrow \pm 1 / 2$, in first order. The first order correction to the NMR frequency of the $m-1 \leftrightarrow m$ transition amounts to $-(m-1 / 2) v_{Q 1}$ with [12]

$$
v_{Q 1}(e q, \eta, \cos \theta, \varphi)=v_{Q}\left[3 \cos ^{2} \theta-1+\eta\left(1-\cos ^{2} \theta\right) \cos 2 \varphi\right],
$$

where

$$
v_{Q}=\frac{3 e^{2} q Q}{4 I(2 I-1) h} .
$$

Here $\eta$ is the asymmetry parameter, $e q$ the largest eigenvalue of the EFG tensor, while $\theta$ and $\varphi$ are the Euler angles defining the direction of the external magnetic field $\boldsymbol{B}_{0}$ with respect to the EFG tensor principle axes system (PAS). Other interactions, e.g. magnetic hyperfine and RKKY interactions, are much smaller than the quadrupole interaction in the case of quasicrystals and significantly affect only the central transition [13]. The shape of the satellite part of the NMR spectrum is thus predominantly determined by the strong quadrupole interaction. The broadening of the satellite part comes from the distribution of quantities $e q$ and $\eta$, which define the shape of the EFG tensor, as well as from the distribution of the EFG tensor PAS orientations that, in turn, produces the distribution of the quantities $\theta$ and $\varphi$. Only the latter, if anisotropic, can cause the NMR spectrum to be orientation-dependent.

[Insert Figure 1 about here.]

In order to test the possible orientation dependence of the ${ }^{27} \mathrm{Al}$ NMR spectrum, we performed experiments on a single-grain $\mathrm{Al}_{69.6} \mathrm{Pd}_{22.1} \mathrm{Mn}_{8.3}$ icosahedral quasicrystal [8]. In another study of the 
same kind [9] no orientation dependence was observed. As the quadrupole-perturbed NMR spectrum turns out to spread over several MHz's, the spectrum was recorded using field-sweep technique and was then recalculated to the frequency scale. The central irradiation frequency was set to $v_{0}\left({ }^{27} \mathrm{Al}\right)=$ 26.134 MHz (corresponding to the centre absorption field $B_{0}=2.35 \mathrm{~T}$ ) and the sample was oriented in the external magnetic field with the 5-fold symmetry axis either perpendicular or parallel to $\boldsymbol{B}_{0}$ (referred to as prp and pll orientations). Both spectra were taken at room temperature and are displayed in Figure 1, a) and b). The narrow, high-intensity peak corresponds to the central transition, while the satellite transitions are joined in the broad »background « line. The satellite parts of both spectra evidently differ. Due to the very long measurement time of the field-sweep experiment, a full rotation pattern of the ${ }^{27} \mathrm{Al}$ spectrum was not conducted. Instead, the angular dependence of the ${ }^{27} \mathrm{Al}$ spectral intensity was measured on a selectively excited frequency interval of $10 \mathrm{kHz}$ at the constant field $0.965 B_{0}$, which corresponded to the point with frequency $1 \mathrm{MHz}$ relative to $v_{0}$ (see Figure $2 \mathrm{a}$ )). The sample was rotated around the axis that lies in the middle between two 2-fold axes and is thus perpendicular to the 5-fold axis. The orientation dependence of the intensity at this point was significant and indicated that the distribution of the EFG tensor orientations in the selected sample was anisotropic.

[Insert Figure 2 about here.]

There is a strong similarity between the spectra from Figures $1 \mathrm{a}$ ) and b) and the spectrum of a hypothetical amorphous material (Figure $1 \mathrm{c}$ )) calculated on te basis of the model of Czjzek et al. [14]. The probability distribution function over the parameters identifying the EFG tensor shape within this spherically isotropic model reads

$$
P(e q, \eta)=\frac{(e q)^{4} \eta}{\sigma^{5} \sqrt{2 \pi}}\left(1-\frac{\eta^{2}}{9}\right) \exp \left[-\frac{(e q)^{2}}{2 \sigma^{2}}\left(1+\frac{\eta^{2}}{3}\right)\right] .
$$

The only free parameter is $\sigma$ and it specifies the width of the distribution function. The distribution over angles $\theta$ and $\varphi$ is isotropic. The probability of finding the site with either cubic $(e q=0)$ or cylindrical $(\eta=0)$ symmetry is zero, in accordance with the situation in icosahedral quasicrystals. Czjzek model was frequently applied to the case of icosahedral quasicrystals [15], always with the tacit assumption of their structure being isotropic on average. In our case the assumption turns out to be invalid. However, the distribution function (3) can be retained and incorporated into the simple auxiliary model, thus somewhat reducing the number of degrees of freedom.

\section{Auxiliary model}

We assume that the distribution of the EFG tensor PAS orientations $f(\Phi, \Theta, \Psi)$ is independent of the distribution $P(e q, \eta)$. Here $\Phi, \Theta$ in $\Psi$ are Euler angles defining the orientation of the EFG tensor PAS with respect to the coordinate system of the sample under study. According to the one-only assumption of the auxiliary model, the distribution $f(\Phi, \Theta, \Psi)$ should have icosahedral symmetry but can otherwise be arbitrary. In search for such a shape of $f(\Phi, \Theta, \Psi)$ that would lead to the observed NMR spectrum orientation-dependence from Figure 2 a), we limited ourselves to the three typical 
distributions that qualitatively covered the whole set of possible distributions with icosahedral symmetry. These are shown in Figure $2 \mathrm{f}$ ), g) and h). The typical distributions are represented by the set of points in the plane $(\cos \Theta, \Psi)$, where each point stands for the direction of that principle axis of the EFG tensor, which corresponds to the largest eigenvalue (referred to as the largest principle axis). In each case the representative points are concentrated around the directions of a certain type of symmetry axes of the sample under study (2-, 3- and 5-fold). The type of the relevant symmetry axes then defines the name of the corresponding distribution (e.g. 2-fold distribution). In the representations from Figure $2 \mathrm{f}$ ), g) and $\mathrm{h}$ ) the distribution over the angle $\Phi$ - which defines the rotation of the EFG tensor around its largest principle axis - remains hidden.

We rotated the sample under study in steps and at each orientation of the sample with respect to the external magnetic field we calculated the directions $(\cos \theta, \varphi)$ of the magnetic field with respect to every EFG tensor PAS of the whole set, joined in the relevant distribution $f(\Phi, \Theta, \Psi)$. The calculated directions form a set of the representative points of some distribution $g(\theta, \varphi)$, which is also independent of $P(e q, \eta)$. By directly multiplying the sets of the representative points of distributions $P(e q, \eta)$ and $g(\theta, \varphi)$, a set of quadruplets $(\cos \theta, \varphi, e q, \eta)$ is obtained, which represent the distribution of the EFG parameters at the atomic sites of the sample under study. For every element of this last set, the NMR frequencies can be calculated according to equation (1) and, taking into account the proper intensity weighting [12], the satellite part of the NMR frequency can be arrived at. In case of each of the typical distributions from Figure $2 \mathrm{f}$ ), g) and $\mathrm{h}$ ) we calculated the orientation dependence of the satellite part of the NMR spectrum and deduced from it the angular dependence of the spectral intensity at the point displaced by $1 \mathrm{MHz}$ with respect to $v_{0}$. The calculated angular dependences are displayed in Figure 2 b), c) and d), respectively.

\section{Conclusions}

The results of the experiment are best reproduced in the case of the 3 -fold distribution $f(\Phi, \Theta, \Psi)$. From the qualitative point of view the correspondence is still good in the case of the 2-fold distribution. In case of the 5-fold distribution the calculated angular dependence behaves just the opposite to the experimental one, having its minima in places of experimental maxima and vice versa. The result of the introduction of the simple auxiliary model is thus twofold. Firstly, we showed that icosahedral symmetry alone can account qualitatively for the observed NMR spectrum orientationdependence. Secondly, we showed that the introduced auxiliary model serves as a filter. On the basis of the measured NMR spectrum orientation-dependence, it can directly differentiate between qualitatively different icosahedral distributions of the EFG tensor orientations. 
Figure 1

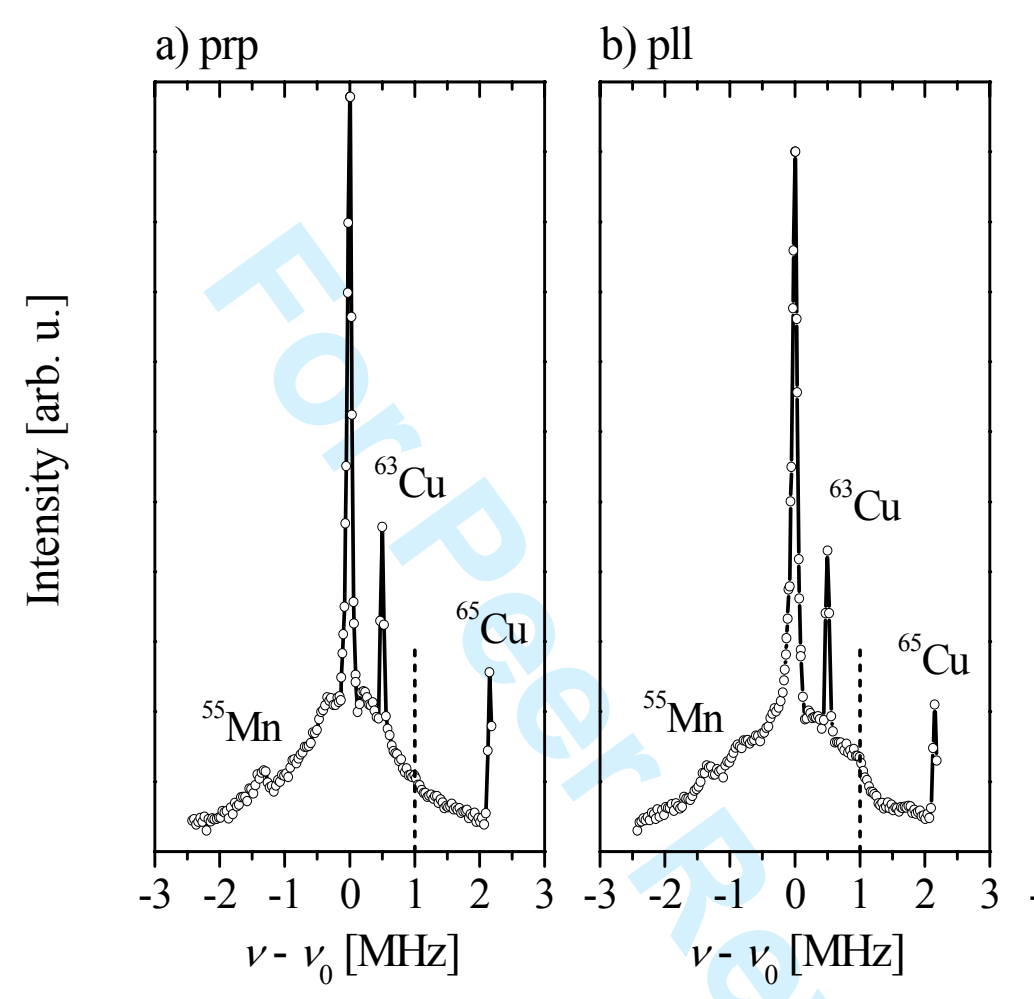

c) iso

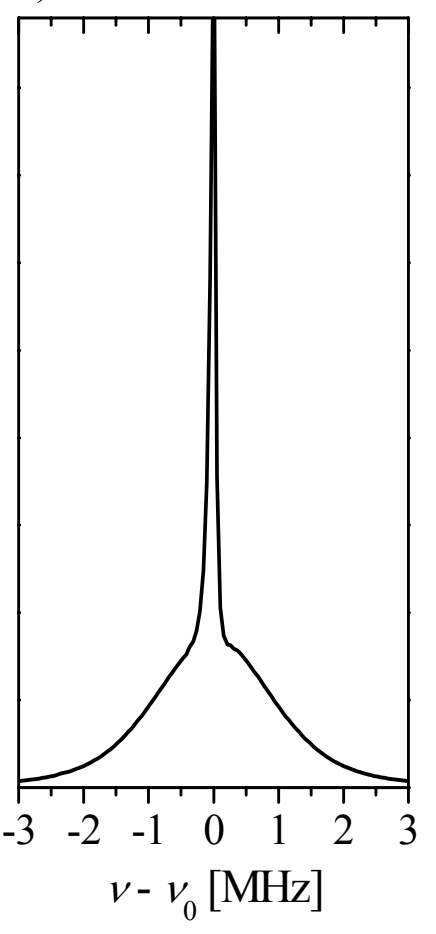


Figure 2
a) $\exp$
b) 2-fold
c) 3-fold
d) 5-fold

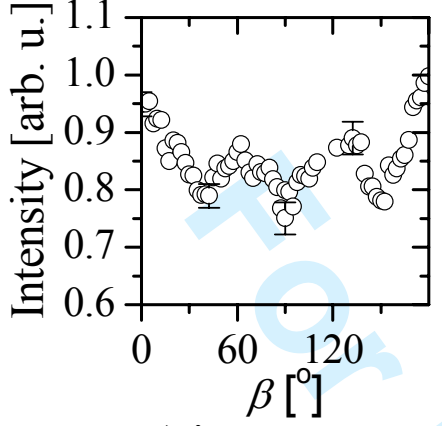

e) iso

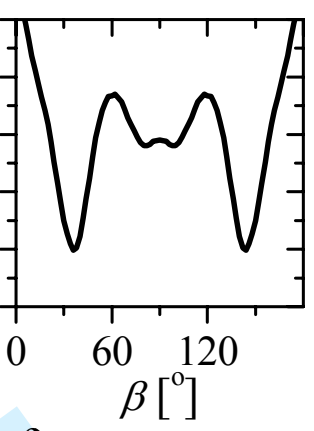

f)

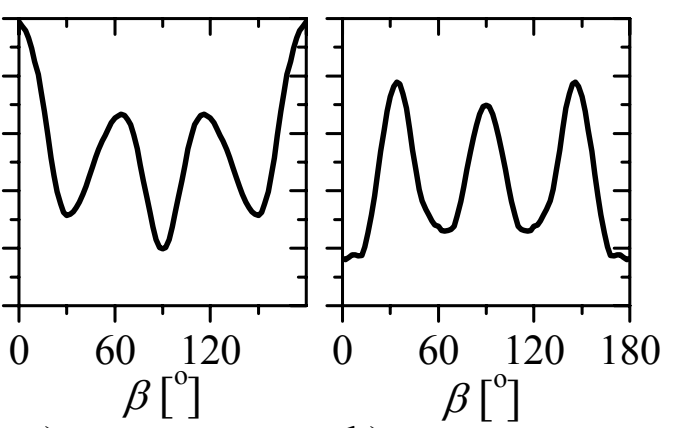

g)

h)

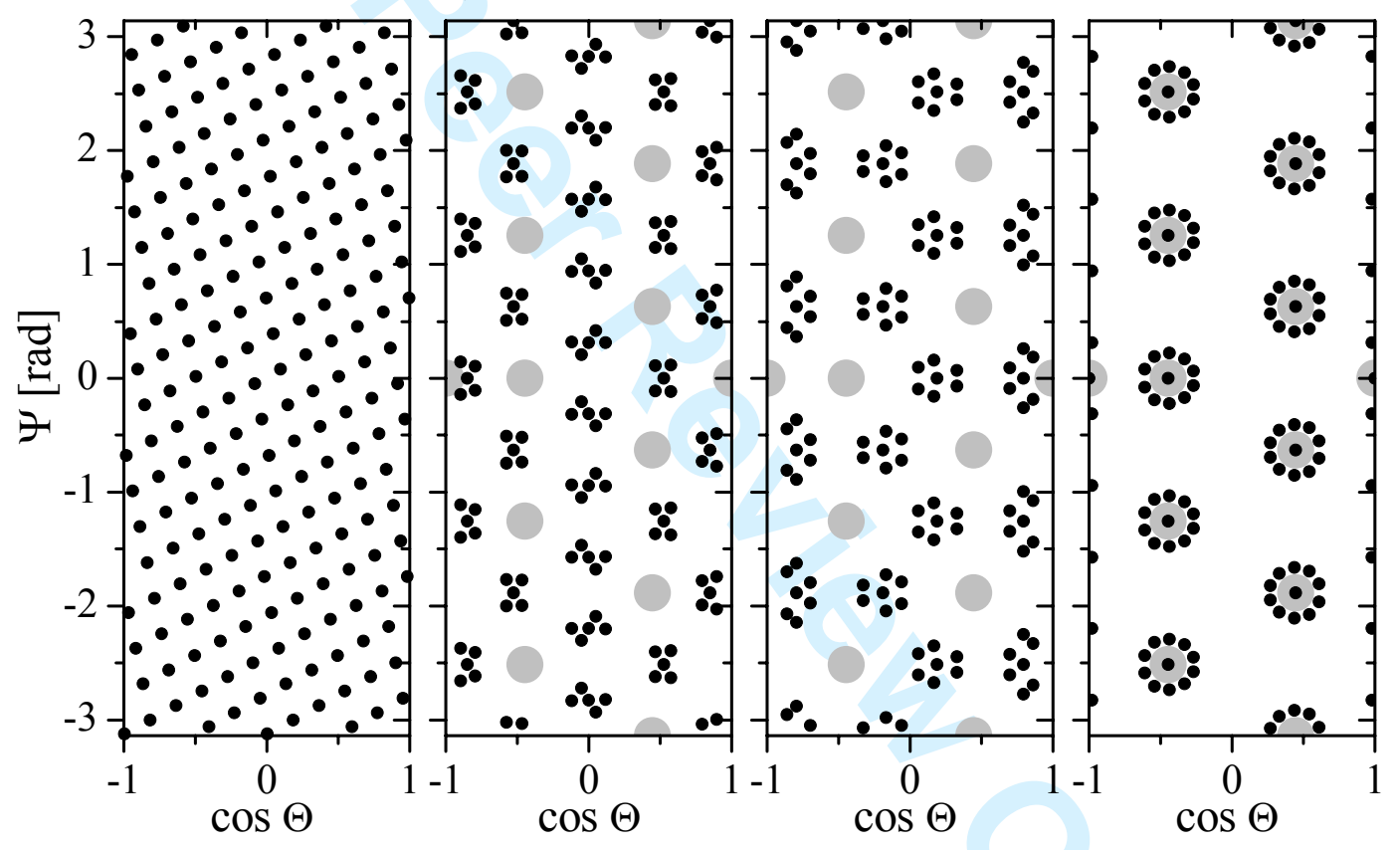




\section{Figure captions}

Figure 1. Orientation-dependent ${ }^{27} \mathrm{Al} \mathrm{NMR}$ spectra of a single-grain icosahedral $\mathrm{Al}_{69.6} \mathrm{Pd}_{22.1} \mathrm{Mn}_{8.3}$ quasicrystal at room temperature $\left(v_{0}\left({ }^{27} \mathrm{Al}\right)=26.134 \mathrm{MHz}, B_{0}=2.35 \mathrm{~T}\right)$. The spectra were recorded using field-sweep technique, and the field was recalculated into frequency units. a) 5-fold axis perpendicular to $\boldsymbol{B}_{0}$. b) 5-fold axis parallel to $\boldsymbol{B}_{0}$. c) $I=5 / 2$ spectrum of the hypothetical amorphous material, which is isotropic on average and can be described by the model proposed by Czjzek et al. (see text). The parameter $\sigma$ is chosen so that the spectrum is close to spectra in a) and b).

Figure 2. a) Experimental angular dependence of the ${ }^{27} \mathrm{Al}$ selectively excited spectral intensity (in frequency window of $10 \mathrm{kHz}$ width) measured at a fixed magnetic field corresponding to the frequency displaced by $1 \mathrm{MHz}$ with respect to $v_{0}$. Here $\beta$ is the angle between the 5 -fold axis and $\boldsymbol{B}_{0}$. The sample was rotated around an axis that lies in the middle between two 2-fold axis and is thus perpendicular to the 5-fold axis. b), c), d) The same angular dependence calculated within the simple auxiliary model described in the text. Three qualitatively different types of the distribution $f(\Phi, \Theta, \Psi)$ of the EFG tensor PAS orientations were considered, i.e. b) 2-fold, c) 3-fold and d) 5-fold (see text), which are displayed in $\mathrm{f}$ ), g) and $\mathrm{h}$ ), respectively. The distribution in e) corresponds to the isotropic amorphous case. In all cases $(\cos \Theta, \Psi)$ defines the direction of that principle axis of the EFG tensor, which corresponds to the largest eigenvalue. Filled gray circles represent the directions of the 5-fold symmetry axes. 


\section{References}

[1] M. Boudard and M. de Boissieu, in Physical Properties of Quasicrystals, edited by Z. Stadnik (Springer-Verlag: Berlin, Heidelberg, New York, 1999), pp. 91-126.

[2] M. Mihalkovič, in Quasicrystals: an Introduction to Structure, Physical Properties, and Applications, edited by J.-B. Suck, M. Schreiber, P. Häusler (Springer-Verlag: Berlin, Heidelberg, New York, 2002), pp. 167-182.

[3] W. Steurer and K. H. Kuo, Acta Crystallographica Section B 46, 703 (1990).

[4] A. I. Goldman and K. F. Kelton, Reviews of Modern Physics 65, 213 (1993).

[5] E. Cockayne, R. Phillips, X. B. Kan, S. C. Moss, J. L. Robertson, T. Ishimasa, and M. Mori, Journal of Non-Crystalline Solids 153\&154, 140 (1993).

[6] A. Quandt and V. Elser, Physical Review B 61, 9336 (2000).

[7] E. S. Zijlstra, J. Kortus, M. Krajčí, Z. M. Stadnik, and S. K. Bose, Physical Review B 69, 094206 (2004).

[8] T. Apih, M. Klanjšek, D. Rau, and J. Dolinšek, Physical Review B 61, 11213 (2000).

[9] A. Shastri, F. Borsa, D. R. Torgeson, J. E. Shield, and A. I. Goldman, Physical Review B 50, 15651 (1994).

[10] A. Shastri, F. Borsa, D. R. Torgeson, and A. I. Goldman, Physical Review B 50, 4224 (1994).

[11] Z. M. Stadnik, T. Takeuchi, N. Tanaka, and U. Mizutani, Journal of Physics: Condensed Matter 15, 6365 (2003).

[12] P. P. Man, in Encyclopedia of Analytical Chemistry, edited by R. A. Meyers (John Wiley and Sons Ltd, Chichester, 2000), pp. 12224-12265.

[13] J. Dolinšek, M. Klanjšek, T. Apih, J. L. Gavilano, K. Giannò, H. R. Ott, J.-M. Dubois, and K. Urban, Physical Review B 64, 024203 (2001).

[14] G. Czjzek, J. Fink, F. Götz, H. Schmidt, J. M. D. Coey, J.-P. Rebouillat, and A. Liénard, Physical Review B 23, 2513 (1981).

[15] R. A. Brand, J. Pelloth, F. Hippert, and Y. Calvayrac, Journal of Physics: Condensed Matter 11, 7523 (1999). 